\title{
Far-infrared photometry of OJ 287 with the Herschel Space Observatory ${ }^{\star} \star \star$
}

\author{
Mark Kidger $^{1}$, Staszek Zola ${ }^{2,3}$, Mauri Valtonen ${ }^{4,5}$, Anne Lähteenmäki ${ }^{6,7,8}$, Emilia Järvelä ${ }^{6,7}$, \\ Merja Tornikoski ${ }^{6}$, Joni Tammi ${ }^{6}$, Alexis Liakos ${ }^{9}$, and Gary Poyner ${ }^{10}$ \\ ${ }^{1}$ European Space Agency, European Space Astronomy Centre, C/ Bajo el Castillo s/n, 28692 Villanueva de la Cañada, Madrid, Spain \\ e-mail: mkidger@scips.esa.int \\ 2 Astronomical Observatory of Jagiellonian University, ul. Orla 171, 30-244 Krakow, Poland \\ ${ }^{3}$ Mt. Suhora Observatory, Pedagogical University, ul. Podchorazych 2, Krakow 30-084, Poland \\ ${ }^{4}$ Finnish Centre for Astronomy with ESO, University of Turku, Turku, Finland \\ 5 Tuorla Observatory, Department of Physics and Astronomy, University of Turku, Turku, Finland \\ ${ }^{6}$ Aalto University, Metsähovi Radio Observatory, Metsähovintie 114, 02540 Kylmälä, Finland \\ 7 Aalto University, Department of Electronics and Nanoengineering, PO Box 15500, 00076 Aalto, Finland \\ 8 Tartu Observatory, Observatooriumi 1, 61602 Töravere, Estonia \\ ${ }^{9}$ Institute for Astronomy, Astrophysics, Space Applications, and Remote Sensing, National Observatory of Athens, Metaxa \& Vas. \\ Pavlou St., 15236 Penteli, Athens, Greece \\ ${ }^{10}$ British Astronomical Association Variable Star Section, 67 Ellerton Road, Kingstanding, Birmingham, UK
}

Received 20 October 2017 / Accepted 3 December 2017

\begin{abstract}
Context. The blazar OJ 287 has shown a $\approx 12$ year quasi-periodicity over more than a century, in addition to the common properties of violent variability in all frequency ranges. It is the strongest known candidate to have a binary singularity in its central engine. Aims. We aim to better understand the different emission components by searching for correlated variability in the flux over four decades of frequency measurements.

Methods. We combined data at frequencies from the millimetric to the visible to characterise the multifrequency light curve in April and May 2010. This includes the only photometric observations of OJ 287 made with the Herschel Space Observatory: five epochs of data obtained over 33 days at 250,350, and $500 \mu \mathrm{m}$ with Herschel-SPIRE.

Results. Although we find that the variability at $37 \mathrm{GHz}$ on timescales of a few weeks correlates with the visible to near-IR spectral energy distribution, there is a small degree of reddening in the continuum at lower flux levels that is revealed by the decreasing rate of decline in the light curve at lower frequencies. However, we see no clear evidence that a rapid flare detected in the light curve during our monitoring in the visible to near-IR light curve is seen either in the Herschel data or at $37 \mathrm{GHz}$, suggesting a low-frequency cut-off in the spectrum of such flares.

Conclusions. We see only marginal evidence of variability in the observations with Herschel over a month, although this may be principally due to the poor sampling. The spectral energy distribution between $37 \mathrm{GHz}$ and the visible can be characterised by two components of approximately constant spectral index: a visible to far-IR component of spectral index $\alpha=-0.95$, and a far-IR to millimetric spectral index of $\alpha=-0.43$. There is no evidence of an excess of emission that would be consistent with the $60 \mu \mathrm{m}$ dust bump found in many active galactic nuclei.
\end{abstract}

Key words. BL Lacertae objects: individual: OJ 287

\section{Introduction}

The blazar OJ 287 is an object of particular interest because of its singular characteristics. It has a redshift of $z=0.306$ based on weak emission lines in its spectrum, as established by Sitko \& Junkkarinen (1985). The commonly accepted paradigm capable of explaining most of the observed radio-loud active galactic nucleus (AGN) properties involves a supermassive black hole surrounded by an accretion disc, feeding a relativistic plasma jet that emits synchrotron and inverse-Compton

\footnotetext{
${ }^{\star}$ Herschel is an ESA space observatory with science instruments provided by European-led Principal Investigator consortia and with important participation from NASA.

$\star \star$ The photometry data (Table 4) is only available at the CDS via anonymous ftp to cdsarc.u-strasbg.fr (130.79.128.5) or via http://cdsarc.u-strasbg.fr/viz-bin/qcat?]/A+A/610/A74
}

radiation. In this scenario, blazars, of which OJ 287 is one of the classic examples, are simply sources in which the relativistic jet is oriented at a small angle to the line of sight. Part of the interest in OJ 287 is that it is believed to be the blazar in which the relativistic jet is most closely aligned with our line of sight. It is the only blazar known to exhibit long-lasting long-period quasi-periodic variability in its light curve.

There have been numerous observations of short-lived periodicity or quasi-periodicity in the light curves of quasars, for example, a well-defined 80-day quasi-periodicity was found in rapid flares in 3C345 between 1965 and 1968 by Kinman et al. (1968), and less clearly, an 800-day quasi-periodicity, also in 3C345, between 1972 and 1976 by Barbieri et al. (1977). There has also been, among others, the detection of a 65-day quasi-periodicity of 3C66a, observed between 1993 and 1998 (Lainela et al. 1999), and various 
periods claimed by Fan et al. (2002) and by Fan et al. (2007) in BL Lac and in the radio in various blazars, respectively. Despite these ephemeral detections, the enduring nature more than a century to date - of the 12-year quasi-periodicity of OJ 287 is unique, as is its demonstrated predictive ability, which has successfully predicted the 1994, 1995, 2005, 2007 (Valtonen et al. 2011), and 2015 (Valtonen et al. 2016) outbursts. A model that successfully explains this observational feature and offers predictions for future light curve behaviour is one in which the blazar central engine contains a binary consisting of two supermassive black holes (SMBHs): a primary of 18.4 $\times 10^{9}$ solar masses with a secondary of $140 \times 10^{6}$ solar masses orbiting their common centre of mass (Valtonen et al. 2010). In this model, the outbursts are caused by the impact of the primary's accretion disk on the secondary twice per orbit at the descending and ascending nodes. The general relativistic orbital precession naturally explains the quasi-periodic nature of the variability of OJ 287 because approximately every five periods, it will cause the observed abrupt shift in the phase of the primary outburst within the phased light curve, as occurred between 1936 and 1948 (Kidger 2000).

\section{Observations}

The Herschel Space Observatory was a $3.5 \mathrm{~m}$ passively cooled Cassegrain telescope in a Lissajous orbit around the L2 point of the Sun-Earth system (Pilbratt et al. 2010). Launched on May 14, 2009, it had a suite of three cryogenic instruments (PACS, SPIRE, and HIFI) that covered the range from $\approx 55-672 \mu \mathrm{m}$ both photometrically, with broadband filters at 70, 100, 160, 250, 350, and $500 \mu \mathrm{m}$, and spectroscopically over its full range of operational wavelengths. Science phase ended with helium depletion on April 29, 2013. Although OJ 287 was not a target approved for observation in Herschel Space Observatory science time, it was recognised in advance of launch that the point-source nature and brightness of blazars in the far-IR made them excellent targets for pointing calibration (Lutz 2008), hence OJ 287 was selected for observation as a suitable bright point-source for spatial calibration observations of the Herschel instruments.

A search was made using the Herschel Science Archive (HSA) for calibration observations of OJ 287 made in standard photometric observing modes. A total of five photometric observations were found, all taken with the SPIRE photometer (Griffin 2010), each giving simultaneous photometry at 250, 350, and $500 \mu \mathrm{m}$. The observations were made on four Operational Days (ODs) between April 20, 2010 and May 23, 2010. All the observations were taken as part of routine instrument calibration under the generic "Calibration_rpspire" programme, but were made in standard observing modes, have high signal-to-noise ratio $(\mathrm{S} / \mathrm{N}$; never <100) and have been declared "science ready" and made public. The journal of observations is shown in Table 1 . OJ 287 is listed in the SPIRE Point Source Catalogue $(250 \mu \mathrm{m}$ source listing) as HSPSC250A_J0854.81+2006.4 (Schulz et al., in prep. ${ }^{1}$ ).

As an illustration of the quality of these data, the postcard from the HSA for a typical OJ 287 observation from those considered here is shown in Fig. 1. The time shown in the postcard header is the local time of the start of the observation. There is a field galaxy $\approx 32$ arcsec north-west of OJ 287, which is clearly resolved at $250 \mu \mathrm{m}$, but increasingly involved with

\footnotetext{
1 The SPIRE Point Source Catalogue and its Explanatory Supplement are available at the https://www.cosmos.esa.int/web/ herschel/spire-point-source-catalogue
}

the blazar at 350 and $500 \mu \mathrm{m}$. This galaxy has a flux lower by an order of magnitude than OJ 287 at 250 and $500 \mu \mathrm{m}$ and a flux lower by a factor of $\approx 7$ than OJ 287 at $350 \mu \mathrm{m}$. We have searched for this field galaxy in the IRAS faint source catalogue, the 2MASS point-source catalogue, the 2MASS faint source catalogue, and the AKARI catalogue, none of which list it, while it does not meet the criteria to be listed in the SPIRE point-source catalogue either.

\section{Results}

\subsection{Herschel data}

Standard pipeline products were retrieved from the HSA. We used the standard recipe given by SPIRE for extracting pointsource photometry from maps using standard Level 2 products from the HSA (Valtchanov 2017). Classic methods of aperture photometry were used to extract the photometry, sampling the sky background in four large apertures around, but wellseparated from, the target to avoid contamination from the tail of the PSF of the target. SPIRE recommend using standard apertures of 44,60 , and $80 \operatorname{arcsec}$ at 250, 350, and $500 \mu \mathrm{m}$, respectively. When the photometry has been measured in these apertures, it must be corrected for two factors: colour correction and light losses. The colour-correction factor is unity for a spectral index, defined as $f_{v} \sim v^{\alpha}$, of $\alpha=-1$ and $<0.2 \%$ for $\alpha=-1.5$, so this factor can be safely neglected for OJ 287 as it will be dominated by other sources of error. The values for the aperture correction to be applied to aperture photometry with SPIRE data are tabulated in Table 5.9 of the SPIRE Handbook (Valtchanov 2017) and were used to generate our final fluxes.

The results are shown in Table 2. Photometric errors for SPIRE photometry are dominated by the uncertainties in the calibration model of $4 \%$, while the reproducibility of SPIRE photometry has been determined to be $1.5 \%$ (Valtchanov 2017). Our photometric error is $\leq 1 \%$ from photon statistics. Thus we estimate that our fluxes have an absolute photometric error of $\approx 6 \%$, but a relative error of $\approx 3 \%$ when all sources of error are considered.

The light curve from these data is shown in Fig. 2. The final observation is significantly fainter than the previous observation in 250 and $350 \mu \mathrm{m}$, but not at $500 \mu \mathrm{m}$. Close inspection of the $500 \mu \mathrm{m}$ observation shows no obvious anomaly in the data.

\subsection{Optical monitoring}

We have three sources of visible monitoring data for this period.

Our first source is the data in $R$ only from the database of long-term monitoring maintained at the Astronomical Observatory of the Jagiellonian University (Cracow, Poland). This consists of 27 observations obtained from 19 nights of photometry in $R$, taken between April 1 and May 28, 2010. Thirteen of the points were taken from Mt. Suhora Observatory, with its $0.6 \mathrm{~m}$ Cassegrain telescope, and five from the Astronomical Observatory of the Jagiellonian University in Cracow, with its $0.5 \mathrm{~m}$ Cassegrain telescope. A further nine observations were obtained in Athens. The median error of these data is 0.023 magnitudes. We note that the penultimate observation from Athens Observatory has an unusually large error bar due to the proximity of the full Moon, but confirmatory data from Cracow were taken less than an hour and a half later, which agree to within $4 \%$. We also have a small amount of data from the British Astronomical Association Variable Star Section archive, obtained in $V$ with the Bradford Robotic Telescope on Tenerife. The raw data, expressed 
Table 1. Journal of observations reported in the text.

\begin{tabular}{lcccc}
\hline \hline Date (UT) & OD & Exposure time & Observing mode & ObsID \\
\hline 20/04/2010 17:30:35 & 341 & $583 \mathrm{~s}$ & Small map & 1342195287 \\
20/04/2010 18:24:59 & 341 & $437 \mathrm{~s}$ & Small map & 1342195290 \\
$21 / 04 / 201002: 55: 58$ & 342 & $1397 \mathrm{~s}$ & Large map & 1342195308 \\
$07 / 05 / 201016: 54: 54$ & 358 & $593 \mathrm{~s}$ & Small map & 1342195867 \\
$23 / 05 / 201014: 22: 11$ & 374 & $1397 \mathrm{~s}$ & Large map & 1342196889 \\
\hline
\end{tabular}

Notes. All these observations were targeted on OJ 287, which was in the centre of the field in all cases. The columns are the date, expressed as the UT mid-time of the exposure; the Operational Day of the mission; the exposure time in seconds; the observing mode; and the Observation, Identifier in the Herschel Science Archive.
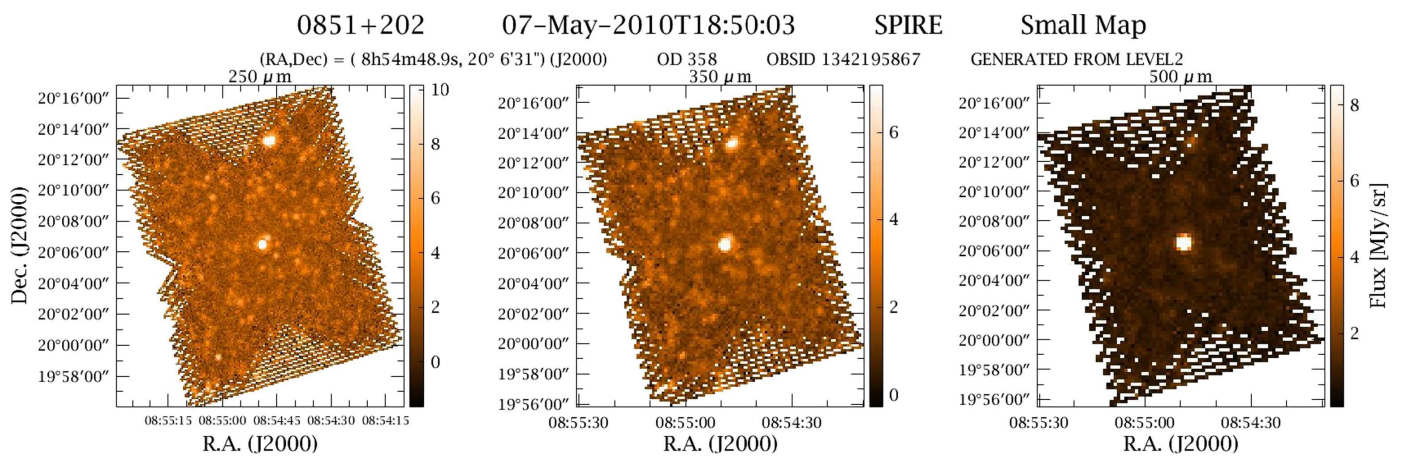

Fig. 1. Postcard image retrieved from the Herschel Science Archive (HSA) of a typical observation from those listed in Table 1, produced by the standard processing pipeline. OJ 287 is at the centre of the field; all other sources are field galaxies. There is a field galaxy $\approx 32$ arcsec north-west of OJ 287, which is clearly resolved at $250 \mu \mathrm{m}$, but increasingly involved with the blazar at 350 and $500 \mu \mathrm{m}$. This galaxy has a flux lower by an order of magnitude than OJ 287 at 250 and $500 \mu \mathrm{m}$ and a flux lower by a factor of $\approx 7$ than OJ 287 at $350 \mu \mathrm{m}$.

Table 2. Reduced Herschel-SPIRE photometry for OJ 287 extracted using the procedure described in the text, applying the appropriate aperture correction to the raw photometry.

\begin{tabular}{lccc}
\hline \hline UT mid-time & $\begin{array}{c}\text { Band } \\
(\mu \mathrm{m})\end{array}$ & $\begin{array}{c}\text { Aperture } \\
(\text { arcsecs })\end{array}$ & $\begin{array}{c}\text { Flux } \\
(\mathrm{Jy})\end{array}$ \\
\hline $20 / 04 / 201017: 30: 34$ & 250 & 44 & 1.09 \\
& 350 & 60 & 1.47 \\
& 500 & 80 & 1.51 \\
$20 / 04 / 201018: 24: 59$ & 250 & 44 & 1.09 \\
& 350 & 60 & 1.38 \\
$21 / 04 / 2010$ 02:55:58 & 500 & 80 & 1.55 \\
& 250 & 44 & 1.02 \\
$07 / 05 / 201016: 54: 54$ & 350 & 60 & 1.31 \\
& 500 & 80 & 1.56 \\
& 250 & 44 & 1.08 \\
$23 / 05 / 201014: 22: 11$ & 350 & 60 & 1.40 \\
& 500 & 80 & 1.59 \\
& 250 & 44 & 0.97 \\
& 350 & 60 & 1.26 \\
& 500 & 80 & 1.52 \\
\hline
\end{tabular}

Notes. Our fluxes have an absolute photometric error of $\approx 6 \%$, but a relative error of $\approx 3 \%$ when all sources of error are considered.

as the difference in magnitude between star 4 of the standard sequence for OJ 287 (Fiorucci \& Tosti 1996) and the blazar were first converted into $B V R$ and then to flux in mJy using the zeromagnitude flux of $4.27 \times 10^{6}, 3.67 \times 10^{6}$, and $2.84 \times 10^{6} \mathrm{mJy}$ for the $B, V$, and $R$ bands, respectively (Zombeck 1990). In this way, when plotting the data, the visible fluxes in mJy are of a similar

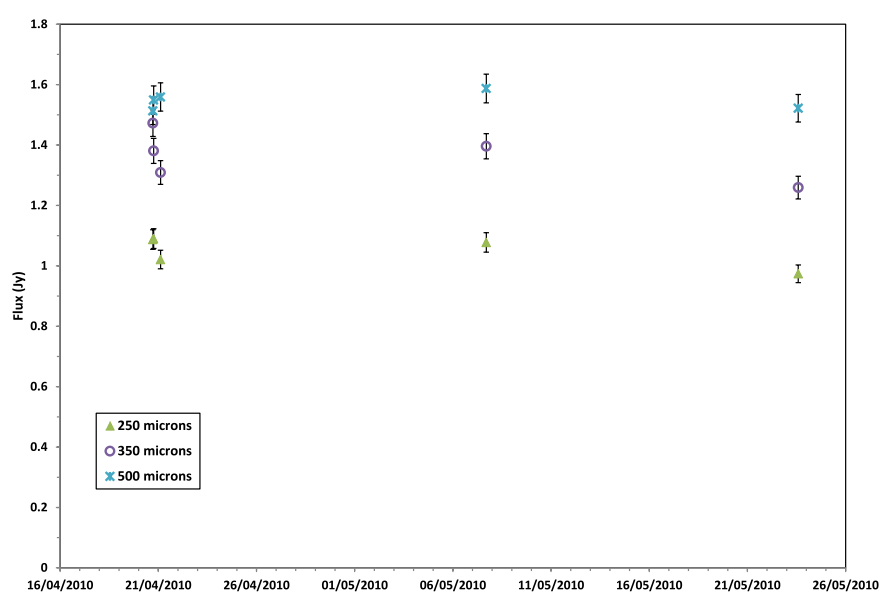

Fig. 2. Light curve of OJ 287 in the Herschel bands from the data presented in the text. There is an evident fading trend particularly between the data collected at mid- and end-May.

order of magnitude to the far-IR fluxes in Jy and can be shown on the same axes in our plots.

Additionally, very high-quality data are publicly available from the SMARTS telescope (Bonning et al. 2012) in $B, V$, and $R$. Typical errors are $<0.01$ magnitudes. Although OJ 287 is not one of the high-cadence SMARTS targets, 26 sets of $B V R$ photometry are available between April 1 and May 31, 2010. SMARTS data were reduced against sequences derived from Landolt standard stars (Landolt 1992). There is some evidence that the SMARTS fluxes are on average $\approx 2 \%$ fainter than the Jagiellonian University fluxes in $R$, indicating a possible small zero-point difference between the two data sets: we have made 
no attempt to plot the data on the same scale as the effect would not be appreciable in our light curves.

\subsection{Near-infrared data}

The SMARTS monitoring programme also obtains high-quality data in the $J$ and $K$ bands in the near-infrared: the median error given by SMARTS for these data is 0.006 magnitudes in $J$ and 0.005 magnitudes in $K$. Three nights have visible, but no infrared photometry, hence $J$ and $K$ data are available on 23 nights in April and May 2010. We note though that there two highly discrepant faint points in $K$ on May 3 and 18 that should be treated with caution.

\subsection{Radio data}

The $37 \mathrm{GHz}$ observations were made with the $13.7 \mathrm{~m}$ diameter Metsähovi radio telescope, which is a radome-enclosed paraboloid antenna situated in Finland. The observations are $\mathrm{ON}-\mathrm{ON}$ observations, alternating the source and the sky in each feed horn. A typical integration time to obtain one flux density data point is between 1200 and $1400 \mathrm{~s}$. The detection limit of the telescope at $37 \mathrm{GHz}$ is of the order of $0.2 \mathrm{Jy}$ under optimal conditions. Data points with an $S / N<4$ are handled as nondetections. The flux density scale is set by observations of DR 21 , with sources NGC 7027, 3C 274, and 3C 84 used as secondary calibrators (Teräsranta et al. 1998). The error estimate in the flux density includes the contribution from the measurement rms and the uncertainty of the absolute calibration.

The temporal coverage is not as high as usual for this normally well-monitored source due to adverse weather conditions and the busy antenna schedule. The combined effect is that there are gaps in the $37 \mathrm{GHz}$ monitoring data, particularly in May 2010. To give context for the Herschel data, all data taken at Metsähovi between April 1, 2010, and May 31, 2010, were used. Sixteen observations were made at Metsähovi between April 5 and May 28, 2010. All were strong detections, with a typical $S / N \approx 30$ and errors in the measured flux of $\approx 3-4 \%$. Of these, 11 points cover the interval between April 5 and 28, when the first three Herschel observations were obtained, and 7 the interval between May 21 and 28, when the final Herschel observation was taken.

\section{Results}

The multifrequency flux curve is shown in Fig. 3. Given the historical range of variability of OJ 287 from $V=12-18$ (Hudec et al. 2013; Takalo et al. 1990) and the demonstrated violent variability at all frequencies at which it has been observed, it is normal to see variations of a factor of 2 in flux over two months, as are seen in the data presented here. We can thus say that the light curve of OJ 287 was active at the time of the Herschel observations, but not especially active.

Both the visible to near infrared and the radio data show significant slow variations on timescales of weeks, although we do not necessarily expect the radio variability to follow the visible to near-IR as the emission in the two ranges could come from different regions of the jet. The most important feature of the light curve during the greater part of April is a steady decline in flux from a maximum at $R \approx 14.0$ at the start of the month. This decline is consistent with the long-term light curve prediction from the binary black hole model, suggesting that the light curve would show a minimum in the first third of 2010 (Sundelius et al. 1997). Although the decline is seen clearly in all bands, except
Table 3. Rate of decline in flux (percent per day) against the band between April 5th and 26th 2010, determined by a least squares fit to the data presented in the text.

\begin{tabular}{cc}
\hline \hline Band & Rate of decline (\%/day) \\
\hline$B$ & 5.0 \\
$V$ & 4.7 \\
$R$ (SMARTS) & 4.6 \\
$R$ (Jagiellonian) & 4.3 \\
$J$ & 3.7 \\
$K$ & 2.6 \\
$37 \mathrm{GHz}$ & 1.4 \\
\hline
\end{tabular}

Notes. There is a clear trend for the spectrum to get redder with declining flux, consistent with known blazar behaviour.

for the far infrared, for which we only have data from Herschel at a single epoch, the slope is much greater at higher frequencies, such that the spectrum becomes significantly steeper and redder with declining flux. This is consistent with normal behaviour in blazars (e.g. Brown et al. 1989). There is only marginal evidence of a decline in the flux in the three Herschel bands over some nine hours on April 20/21. The effect of reddening of the continuum as the blazar becomes fainter is quantified in Table 3.

The second feature of the light curve is the flare between April 26 and May 11 that is seen in $B, V, R$, and $J$, but is less clear in $K$ and not visible in the data at $37 \mathrm{GHz}$ (see Fig. 4). Such flares are a typical feature of the light curve of OJ 287, generally last 10-15 days, and have amplitudes of a few tens of percent in flux. In the $R$-band data we see the flux rise from $2.9 \mathrm{mJy}$ to $4.1 \mathrm{mJy}$ ( $40 \%$ increase) in seven days, followed by a decline on a similar timescale. Exactly the same amplitude (40\%) is seen in $B$ and in $R$, whereas in $J$, the flux increase is $\approx 30 \%$ and in $K$, it is $\approx 10-15 \%$. The data at $37 \mathrm{GHz}$ cover the initial part of the rise, but show no evidence of any increase in flux. Similarly, we have Herschel data at the peak of the flare that show approximately the same flux level as pre- or post-flare, with no clear evidence of an increase in flux. Hence, it appears that the spectral index in the visible range does not change during the flare, but the spectral energy distribution (SED) of the flare shows a low-frequency cut-off in the near-infrared.

The final feature of the light curve is a period of approximately stable flux in all bands between mid-May and the end of the interval covered by the light curve on May 31.

All the source data used in this paper are listed in Table 4 and are available at the CDS, which lists the following information: cols. 1-4: Modified Julian Day (MJD), $37 \mathrm{GHz}$ flux (Jy), error (Jy) and observatory; cols. 5-12: MJD, $500 \mu \mathrm{m}$ flux (Jy) and error (Jy), $350 \mu \mathrm{m}$ flux (Jy) and error (Jy), $250 \mu \mathrm{m}$ flux (Jy) and error (Jy) and observatory; cols. 13-16: MJD, $K$ magnitude, $K$ magnitude error and observatory; cols. 17-20: MJD, $J$ magnitude, $J$ magnitude error and observatory; cols. 21-24: MJD, $R$ magnitude, $R$ magnitude error and observatory; cols. 25-28: MJD, $V$ magnitude, $V$ magnitude error, and observatory; and cols. 29-32: MJD, $B$ magnitude, $B$ magnitude error and observatory. We can use these multifrequency data to construct the SED at different epochs of our coverage. As there are three Herschel observations separated by approximately nine hours on April 20/21, 2017, we averaged these observations. Similarly, in the absence of evidence of rapid high-amplitude variability, where we do not have data in other bands that are exactly simultaneous with the Herschel data, we averaged the nearest data before and after the Herschel observations to give the best estimate of the flux at the epoch of the Herschel observation; but even 


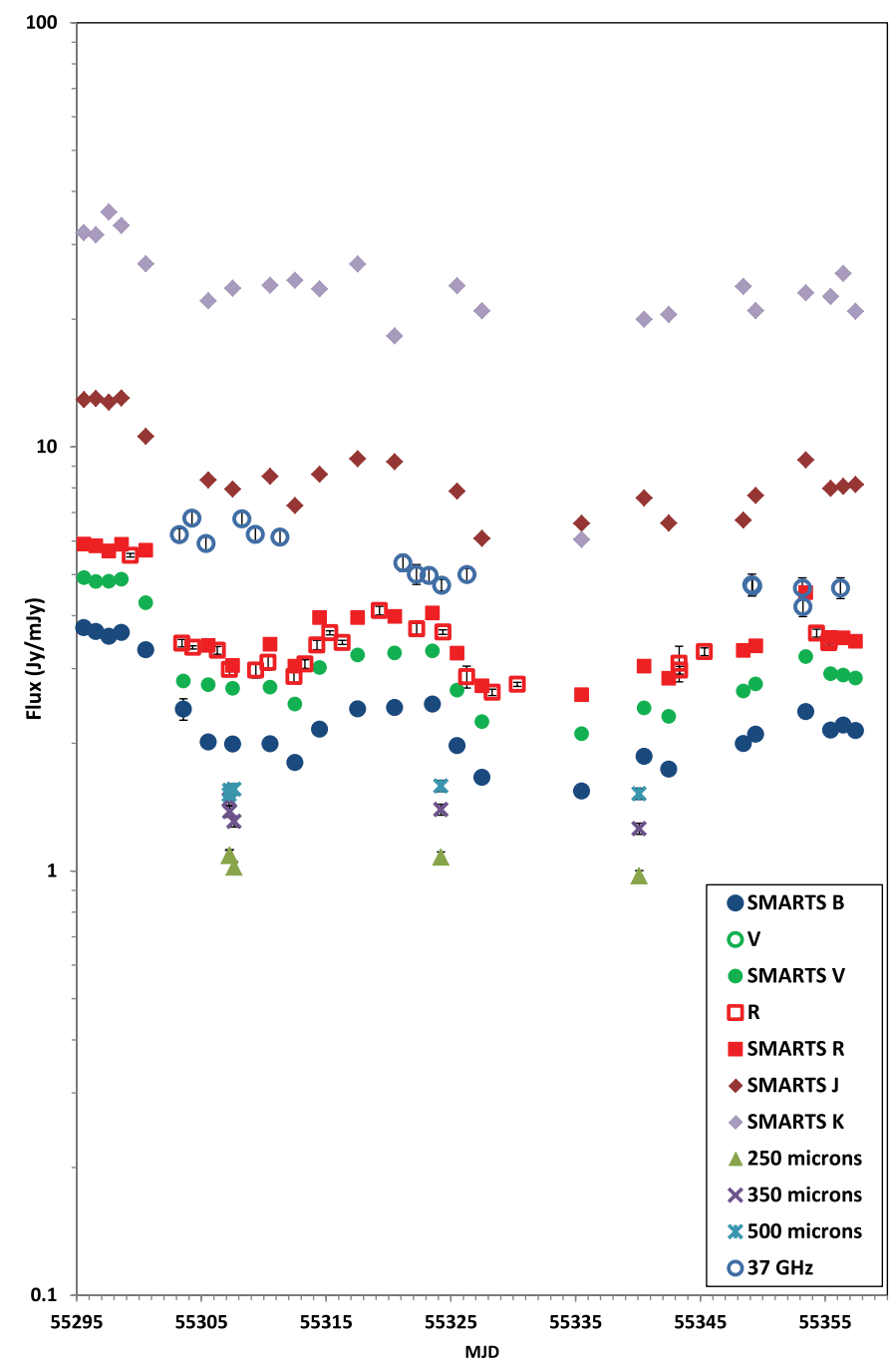

Fig. 3. Flux curve of OJ 287 for April-May 2010. The Herschel-SPIRE data at 250,350 , and $500 \mu \mathrm{m}$ are shown alongside radio data from Metsähovi at $37 \mathrm{GHz}(8 \mathrm{~mm})$, the visible data in the $R$ band from the Jagiellonian University archive, and SMARTS data in $B V R J K$. For clarity, the data in the visible and near-infrared are plotted in mJy so that they can be shown on the same scale as the radio and far-IR data (plotted in Jy). In many cases, the quoted error bars are smaller than the point size. No offset has been applied to bring the two main datasets in $R$ onto the same scale, although there is evidence that on average, the SMARTS fluxes are $\approx 2 \%$ fainter than the Jagiellonian University/Mt. Suhora fluxes.

so, no Metsähovi data are close enough to our second epoch of Herschel observations to be useful in defining the SED. The resulting SEDs are shown in Fig. 5.

We note that the Galactic latitude of OJ 287 is quite high $\left(b^{I I}=35^{\circ} .8\right)$. The calculated interstellar extinction for OJ 287 given for the object by NED, based on the values of Schlafly \& Finkbeiner (2011), calculated from IRAS/ISSA and Planck/DIRBE, is $A_{\lambda}(V)=0.077$ magnitudes, with corresponding values of $A_{\lambda}(R)$ and $A_{\lambda}(K)$ of 0.061 and 0.008 magnitudes, respectively. Thus the de-reddening correction for Fig. 5 is not appreciable.

\section{Discussion}

Our observations cover a period of only moderate activity of the blazar, limiting our ability to investigate possible changes in the

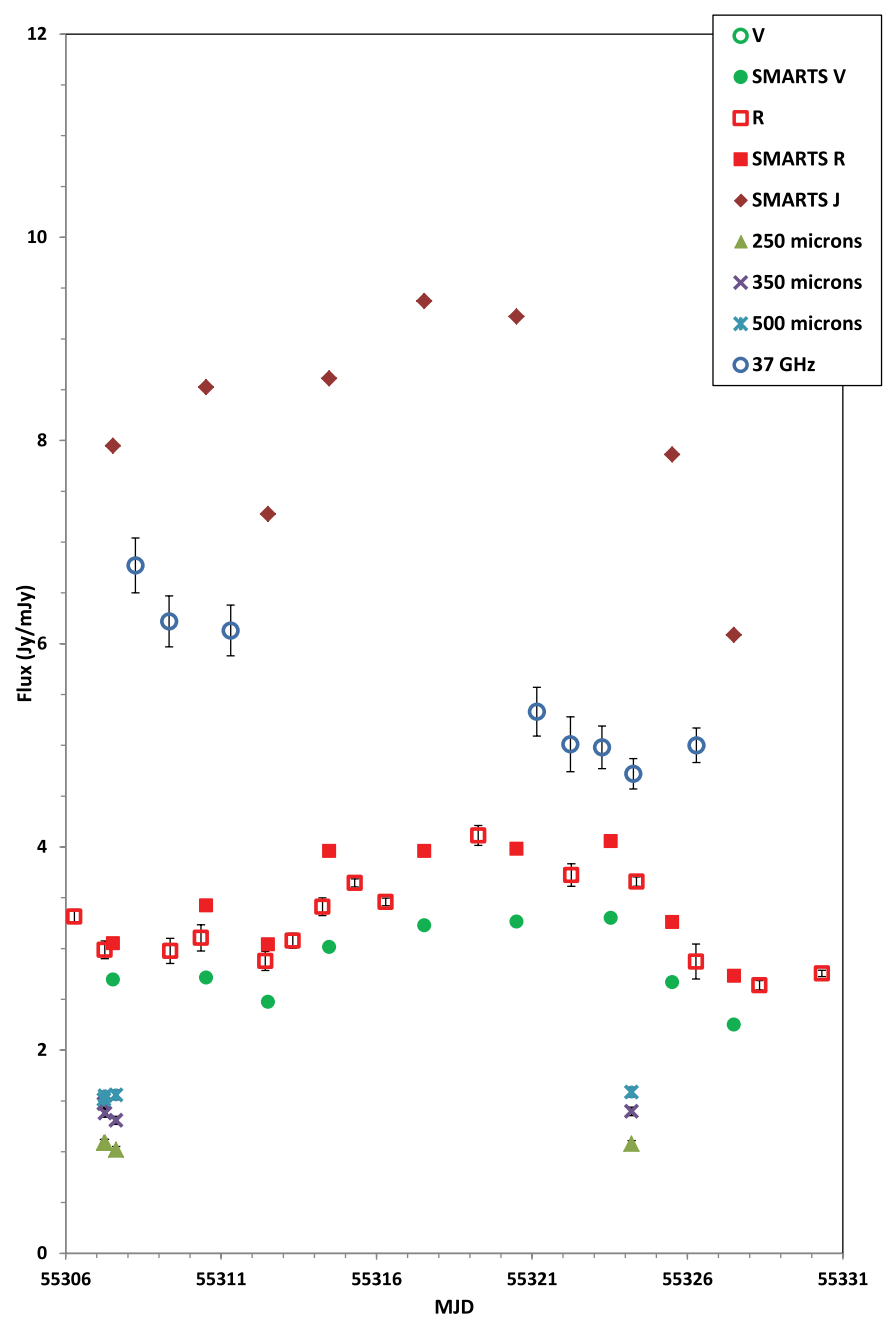

Fig. 4. Flux curve of OJ 287 for the flare in late April and early May 2010 on an expanded scale. The flux in $R$ increases by $40 \%$ in seven days. For clarity, the $K$ data are neglected because they have more dispersion and do not show the flare clearly. Both the Herschel data and the $37 \mathrm{GHz}$ data overlap the flare, with the Herschel data at the peak of the flare, but there is no clear evidence of an increase in flux in either frequency range at this time.

visible to far-IR SED. We see that the far-IR to millimetric spectrum is clearly flatter than the far-IR to visible and that the overall SED can be split into two components, each with approximately constant spectral index: the optical to far-IR spectral index at all three epochs of Herschel data is $\alpha=-0.95$ between $250 \mu \mathrm{m}$ and $B$; for the two epochs with simultaneous data at $37 \mathrm{GHz}$ and in the far-IR, we find a spectral index between $37 \mathrm{GHz}$ and $250 \mu \mathrm{m}$ of $\alpha=-0.43$. The radio spectrum of OJ 287 shows a turnover at $\approx 50 \mathrm{GHz}$ and has been investigated in detail at $1.1-857 \mathrm{GHz}$ by Ade et al. (2016), who combined multi-epoch survey data from the Planck satellite with ground-based radio telescopes, finding that in early 2010, OJ 287 had shown the largest flare ever observed in $37 \mathrm{GHz}$. The authors note that the turnover frequency was highest at the highest level of $37 \mathrm{GHz}$ flux, consistent with the predictions of the shock model. The start of our monitoring coincides with the final phase of the return to quiescence of this large flare.

There are numerous studies of the variability of the spectral index of OJ 287 between the far-IR and UV, mostly limited to the optical range. A slow reddening of the $(B-V)$ spectral index from 0.36 to 0.70 was found after the 1972 outburst 


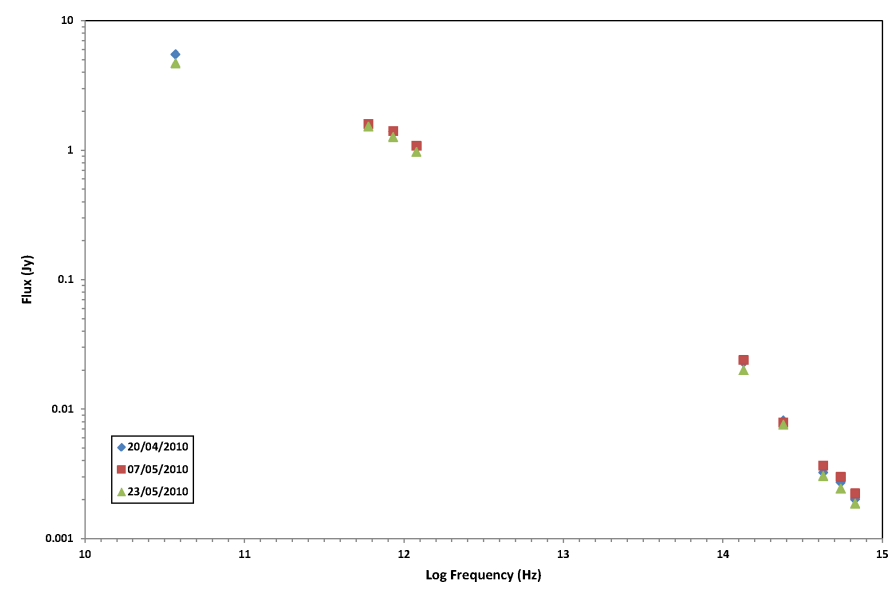

Fig. 5. SEDs for the three epochs of Herschel observations.

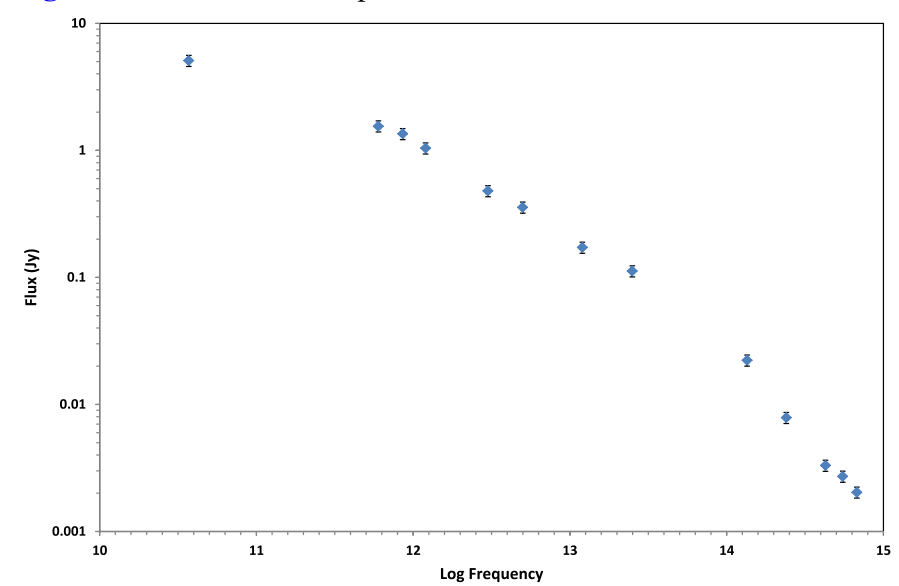

Fig. 6. Averaged SED for OJ 287 from the data in Fig. 5, including data from the IRAS faint source catalogue, scaled by $\times 0.4$ to bring them to the same level as the interpolation of the Herschel and visible to near-IR data. A nominal $10 \%$ error bar has been plotted on each datum. There is no evidence of a $60 \mu \mathrm{m}$ bump from possible dust emission from the host galaxy.

(Takalo \& Silanpää 1989). After the 1983 outburst, the reddening of the colours was faster, but the final $(B-V)$ colour the same as after the 1972 outburst, with $(B-V)=0.46$ at peak of outburst and $(B-V)=0.70$ at post-outburst minimum. Similar conclusions are reached by more recent studies (Zhang \& Xie 1996; Zheng et al. 2008): the latter found a best period of 11.96 years in the spectral index variations in the visible, consistent with the quasi-periodicity of the outbursts. A correlation of spectral index with flux level after the 1983 outburst was found to extend into the infrared (Gear et al. 1986); both the change in the spectral index and the reported periodicity are consistent with the model in which there are massive injections of relativistic electrons in an outburst that then decay slowly radiatively (Marscher \& Gear 1985; Brown et al. 1989). This radiative decay model, or shocked jet, has been developed further by other authors (Kirk et al. 1998; Masichiadis \& Kirk 2002) and has been used by Sundelius et al. (1997) for long-term modelling of the behaviour of the light curve from mass accretion flows. Alternative explanations for the correlation of spectral index with flux level have been offered by Villata et al. (2004), who proposed based on observations of BL Lac that this reddening might be explained by a Doppler factor variation on a convex spectrum. They found that the variability of BL Lac and its colour changes can be explained by a combination of two emission components plus the constant background flux of the host galaxy. We also refer to the extensive discussion of alternative models to explain radio variability by Ade et al. (2016).

The host galaxy for OJ 287 has been weakly detected at $2.2 \mu \mathrm{m}$ (Wright et al. 1998) and is consistent with being a giant elliptical with an absolute magnitude in $K$ in the range of -28.7 to -29.1 , similar to the host galaxy of other blazers. Nilsson (2017, priv. comm.) found a somewhat fainter host galaxy magnitude from observations in $K$ with the Nordic Optical Telescope at the Observatorio del Roque de los Muchachos (La Palma), although, given the modelling uncertainties of the brightness profile, these results are not necessarily incompatible. Other authors found evidence from XMM-Newton observations of absorption from the host galaxy with a hydrogen column density of $\approx 9.3 \times 10^{20} \mathrm{~cm}^{-2}$, which is a factor of $\sim 3$ higher than observed within our Galaxy (Valtonen et al. 2012). In OJ 287, the contrast between the blazar emission and the host galaxy is also too great for the host galaxy to affect the slope of the SED in the visible or near-IR. Unlike for other less strongly beamed sources such as BL Lac itself, it is generally assumed that the colour index of OJ 287 is constant on time scales of weeks or a few months (e.g. Zola et al. 2016), even when OJ 287 is at unusually faint epochs. Here we present evidence, however, that although the slow decline in flux is correlated at frequencies between $37 \mathrm{GHz}$ and $B$, the slope of the decline increases at higher frequency, suggesting a significant reddening of the spectrum on a timescale of a few weeks, although the effect is too small to be detectable in just the $(B-V)$ colour index that was used for previous studies.

Any potential $60 \mu \mathrm{m}$ peak of dust emission from the host galaxy of OJ 287 would be redshifted to $\approx 80 \mu \mathrm{m}$ in OJ 287 , so that we will still be close to the peak of any dust emission from the host galaxy even at $250 \mu \mathrm{m}$ (Guiderdoni et al. 1998). To determine whether there is any evidence of a possible $60 \mu \mathrm{m}$ bump, we also examined the complementary data from IRAS. These data were taken during 1983, when OJ 287 was at a relatively high state due to the 1983 outburst. Although OJ 287 is not included in the IRAS point-source catalogue, it is included in the IRAS faint source catalogue (Moshir et al. 1990) as IRAS F08519+2017. The quality flag for the data is " 3 " (good quality) at 12,25 , and $60 \mu \mathrm{m}$ and " 2 " at $100 \mu \mathrm{m}$, with no cirrus confusion. We multiplied the IRAS FSC fluxes by 0.4 to bring them to the same level as the extrapolation of the Herschel and the visible to near-IR data, and in other bands, we took the mean of the data plotted in Fig. 5. The result is shown in Fig. 6: there is no evidence of a $60 \mu \mathrm{m}$ bump due to the host galaxy, or alternatively, to contamination from the nearby foreground galaxy, and the IRAS data appear to be a smooth continuation of the Herschel-SPIRE SED, although the Herschel data were taken at a rather lower state of OJ 287 than the IRAS data.

In addition to a contribution from the host galaxy, the possibility of detecting torus emission in the mid-IR of BL Lac objects has also been contemplated and was studied by Plotkin et al. (2012) using WISE data: no such emission was detected, and many of the objects in their sample were found to be so strongly beamed that the jet emission completely dominated the AGN IR spectrum, as we find is the case for OJ 287.

\section{Conclusions}

We can enumerate our main conclusions from this study as the following:

1. We have presented the only Herschel photometric observations of the blazar OJ 287. These show the 
blazar in a moderately low state. The data were combined with quasi-simultaneous data at radio, near-IR, and optical frequencies to investigate the SED and its behaviour over four decades of frequency measurements.

2. We see correlated slow variability from radio to optical in the form of a slow fading over three weeks in April 2010. The fading rate shows a strong correlation with frequency; the SED becomes steeper as OJ 287 fades, consistent with models.

3. In contrast, we do not see clear evidence of the rapid flares that are characteristic of the visible light curve of OJ 287 and of other blazars at lower frequencies, suggesting that the emitting mechanism for rapid flares has a low-frequency cutoff.

4. Combination of Herschel data with historical IRAS data shows no evidence of a $60 \mu \mathrm{m}$ bump in the spectrum, either from the blazar or from the nearby foreground galaxy to the north-east. We conclude that the contrast with the bright blazar nucleus is too great even at this relatively faint magnitude to permit detection of possible dust emission from the host galaxy.

Acknowledgements. The Herschel spacecraft was designed, built, tested, and launched under a contract to ESA managed by the Herschel/Planck Project Team by an industrial consortium under the overall responsibility of the prime contractor Thales Alenia Space (Cannes), and including Astrium (Friedrichshafen) responsible for the payload module and for system testing at spacecraft level, Thales Alenia Space (Turin) responsible for the service module, and Astrium (Toulouse) responsible for the telescope, with in excess of a hundred subcontractors. SPIRE has been developed by a consortium of institutes led by Cardiff University (UK) and including Univ. Lethbridge (Canada); NAOC (China); CEA, LAM (France); IFSI, Univ. Padua (Italy); IAC (Spain); Stockholm Observatory (Sweden); Imperial College London, RAL, UCL-MSSL, UKATC, Univ. Sussex (UK); and Caltech, JPL, NHSC, Univ. Colorado (USA). This development has been supported by national funding agencies: CSA (Canada); NAOC (China); CEA, CNES, CNRS (France); ASI (Italy); MCINN (Spain); SNSB (Sweden); STFC, UKSA (UK); and NASA (USA). This paper has made use of HSpot \& HIPE, which are joint developments by the Herschel Science Ground Segment Consortium, consisting of ESA, the NASA Herschel Science Center, and the HIFI, PACS and SPIRE consortia. This paper has made use of up-to-date SMARTS optical/near-infrared light curves that are available at the url: www . astro.yale.edu/smarts/glast/home.php. This work was partly supported by the NCN grant No. 2013/09/B/ST9/00599.

\section{References}

Ade, P. A. R., Aghanim, N., Aller, H. D., et al. 2016, A\&A, 596, A106 Barbieri, C., Romano, G., di Serego, S., \& Zambon, M. 1977, A\&A, 59, 419
Bonning, E., Urry, C. M., Bailyn, C., et al. 2012, ApJ, 756, 13 Brown, L. M. J., Robson, E. I., Gear, W. K., et al. 1989, ApJ, 340, 129

Fan, J. H., Lin, R. G., Xie, G. Z., et al. 2002, A\&A, 381, 1

Fan, J. H., Liu, Y., Yuan, Y. H., et al. 2007, A\&A, 462, 547

Fiorucci, M., \& Tosti, G. 1996, A\&AS, 116, 403

Gear, W. K., Robson, E. I., \& Brown, L. M. J. 1986, Nature, 324, 546

Griffin, M. J., Abergel, A., Abreu, A., et al. 2010, A\&A, 518, L3

Guiderdoni, B., Hivon, E., Bouchet, F. R., \& Maffei, B. 1998, MNRAS, 295, 877

Hudec, R., Basta, M., Pihajoki, P., \& Valtonen, M. 2013, A\&A, 559, A20

Kidger, M. R. 1989, A\&A, 226, 9

Kidger, M. R. 2000, AJ, 119, 2053

Kinman, T. D., Lamla, E., Ciurla, T., et al. 1968, ApJ, 152, 357

Kirk, J. G., Rieger, F. M., \& Mastichiadis, A. 1998, A\&A, 333, 452

Lainela, M., Takalo, L. O., Sillapää, A., Pursimo, T., et al. 1999, ApJ, 521, 561

Landolt, A. U. 1992, AJ, 104, 340

Lehto, H. J., \& Valtonen, M. J. 1996, ApJ, 460, 207

Lutz, D. 2008, Use of blazars for PACS spatial calibration, PACS Instrument Control Centre Technical Note, PICC-MA-TN-024, https://www. cosmos.esa.int/documents/12133/996891/Use+ of+Blazars+for+PACS+spatial+calibration

Marscher, A. P., \& Gear, W. K. 1985, ApJ, 298, 114

Mastichiadis, A., \& Kirk, J. G. 2002, Publ. Astron. Soc. Aust., 19, 138

Moshir, M., Kopan, G., Conrow, T., et al. 1990, BAAS, 22, 1325

Pilbratt, G. L., Riedinger, J. R., Passvogel, T., et al. 2010, A\&A, 518, L1

Plotkin, R. M., Anderson, S. F., Brandt, W. N., et al. 2012, ApJ, 745, L27

Schlafly, E. F., \& Finkbeiner, D. P. 2011, ApJ, 737, 103

Sitko, M. L., \& Junkkarinen, V. T. 1985, PASP, 97, 1158

Sundelius, B., Wahde, M., Lehto, H. J., \& Valtonen, M. J. 1997, ApJ, 484, 180

Takalo, L. O., \& Sillanpää, A. 1989, A\&A, 218, 45

Takalo, L. O., Kidger, M. R., de Diego, J. A., Sillanpää, A., Piirola, V., \& Teräsranta, H. 1990, A\&AS, 83, 459

Teräsranta, H., Tornikoski, M., Mujunen, A., et al. 1998, A\&AS, 132, 305

Valtchanov, I. 2017, The SPIRE Handbook, HERSCHEL-HSC-DOC0798, https://wWw.cosmos.esa.int/documents/12133/1035800/ The+Herschel+Explanatory+Supplement\%2C+Volume+IV+-+THE+ SPECTRAL+AND+PHOTOMETRIC+IMAGING+RECEIVER+\%28SPIRE\%29

Valtonen, M. J., Lehto, H. J., Nilsson, K., et al. 2008a, Nature, 452, 851

Valtonen, M. J., Zola, S., Ciprini, S., et al. 2008b, MNRAS, 385, 823

Valtonen, M. J., Mikkola, S., Merritt, D., et al. 2010, ApJ, 709, 725

Valtonen, M. J., Lehto, H. J., Takalo, L. O., \& Sillanpää, A. 2011, ApJ, 729,

Valtonen, M. J., Ciprini, S., \& Lehto, H. J. 2012, MNRAS, 427, 77

Valtonen, M. J., Zola, S., Ciprini, S., et al. 2016, ApJ, 819, L37

Villata, M., Raiteri, C. M., Kurtanidze, O. M., et al. 2004, A\&A, 421, 103

Wright, S. C., McHardy, I. M., \& Abraham, R. G. 1998, MNRAS, 295, 799

Zhang, Y. H., \& Xie, G. Z. 1996, A\&AS, 119, 199

Zheng, Y. G., Zhang, X., Bi, X. W., et al. 2008, MNRAS, 385, 823

Zheng, W., Telting, J., Saario, J., et al. 2016, ApJ, 819, L37

Zola, S., Valtonen, M., Bhatta, G., Goyal, A., et al. 2016, Galaxies, 4, 41

Zombeck, M. V. 1990, Handbook of Astronomy and Astrophysics (Cambridge, UK: Cambridge University Press) 